\title{
COMMUNICATION OR COGNITION?: A MINIMAL COMPUTATIONAL MODEL OF EVOLUTIONARY INTERACTIONS BETWEEN INDIVIDUAL AND SOCIAL LEARNING
}

\author{
Masahiko Higashi $^{* 1}$, Reiji Suzuki ${ }^{1}$, and Takaya Arita ${ }^{1}$ \\ *Corresponding Author: higashi@alife.cs.is.nagoya-u.ac.jp \\ ${ }^{1}$ Graduate School of Informatics, Nagoya University, Japan
}

\section{Introduction}

Animals including humans adapt to the environment by 2 different mechanisms working on 2 levels, evolution and learning, a population level mechanism and an individual level mechanism, respectively. Learning can further be classified into individual learning and social learning ( $I L$ and $S L$, hereafter). Our purpose is to understand how complex systems, specifically language emerged through the evolutionary interaction between IL and SL by using an agent-based model. Language is a communication tool but also a cognitive tool while most evolutionary scenarios see it as the former (Reboul, 2015). Indeed, in the brain, utilizing language-related circuits, some form of linguistic knowledge is linked to the external world by producing/perceiving sounds and gestures, and at the same time, is connected to the inner mental world composed of concepts, intentions and reasoning (Berwick et. al., 2013), regardless whether there is a shared computational core (e.g. Strong Minimalist Thesis (Chomsky, 2000)).

We assume the fitness function represents the 2 components of selection. One is the directional component $(D C$, hereafter) that drives the evolution of fundamental traits underlying the whole linguistic activities. For DC, we do not use a simple unimodal function but a minimal fitness function representing a multimodal fitness landscape with linguistic levels. A linguistic level represents a fitness value expected when communication succeeds, corresponding to the number of traits involved in the trait interaction. The basic idea is as follows. In general, language ability regardless of cognitive or communicative is based on multiple subordinate traits, and its mutual interactions are nonlinear. We can further assume that the more adaptive the trait set is, the more inter-trait 
interactions are required. In other words, there is a trade-off between adaptivity of individuals and the strength of the epistatic/nonlinear interactions among phenotypes. We represent this idea by using a simple rugged fitness function (Suzuki \& Arita, 2007). The other is the positive frequency-dependent component ( $P F C$, hereafter). It has been pointed out that mutations in grammar cannot be beneficial because a mutant's peers might not understand her (Pinker \& Bloom, 1990; Glackin, 2010). To represent the collective adaptivity of language, that is related with not only communicative but also cognitive aspects, we simply define PFC of an individual as the ratio of the others with the same linguistic level. When considering the adaptive evolution, it would be plausible to consider either aspect (cognition or communication) of language evolved under the selection with both components. We hypothesize that the evolution of language as a communication tool depended more on PFC than DC, comparing with that of language as a cognitive tool, while we carefully distinguish between proposing language evolved for $\mathrm{A}$, and proposing language evolved as a system of A (or just as being used in A). We thus can change indirectly the proportion of the both aspects by changing the proportion of both components.

We assume an intergenerationally overlapped population in which each performs IL based on trial-and-error of phenotypic changes and SL based on imitation of phenotypes from the most adaptive individual. We evolved the initial phenotypic values, their plasticity and a SL rate via fitness proportional selection. First, we investigated the behavior of the model without PFC. We found that both IL and SL worked cooperatively, and facilitated the evolution of cognitive aspect, enabling the population to cross a fitness valley repeatedly. In this process, IL enabled an individual to find new adaptive phenotypes through its trial-and-error process. SL enabled the whole population to share such adaptive phenotypes, which brought about the genetic assimilation of acquired phenotypes. SL further facilitated an acquisition of more adaptive phenotypes through IL by increasing the genetic diversity of the population. As the proportion of PFC increased, such a cooperative evolutionary process was more significantly retarded, and the population tended to converge to a lower peak. We can discuss possible scenarios of language evolution based on the results. A basic scenario might be the following. As the population size increased, the chance of interaction among members tended to increase. Therefore, we can assume that it led to an increase in the selection pressure caused by PFC, which tended to decelerate the evolution.

\section{Acknowledgements}

This work was supported by MEXT/JSPS KAKENHI Grant Number \#4903, JP17H06383. 


\section{References}

Berwick, R. C., Friederici, A. D., Chomsky, N., \& Bolhuis, J. J. (2013). Evolution, brain, and the nature of language. Trends in cognitive sciences, 17(2), 89-98.

Chomsky, N. (2000). Minimalist inquiries: The framework. In: Martin, R., Michaels, D., Uriager - eka, J., editors. Step by step: Essays on minimalist syntax in honor of Howard Lasnik (pp. 89-155). Cambridge (Massachusetts): MIT Press.

Glackin, S. N., (2010). Universal grammar and the Baldwin effect: a hypothesis and some philosophical consequences. Biology and Philosophy, 26(2), 201222.

Pinker, S., \& Bloom, P. (1990). Natural language and natural selection. Behavioral and Brain Sciences, 13, 707-784.

Reboul, A. C. (2015). Why language really is not a communication system: a cognitive view of language evolution. Frontiers in Psychology, 6, Article ID: 1434.

Suzuki, R., \& Arita, T. (2007). Repeated occurrences of the Baldwin effect can guide evolution on rugged fitness landscapes. In Proceedings of the First IEEE Symposium on Artificial Life (IEEE-Alife'07), 8-14. 